\title{
The importance of patient participation in measuring rheumatoid arthritis flares
}

\section{Clifton 0 Bingham III, ${ }^{1}$ Rieke Alten, ${ }^{2}$ Maarten P de Wit ${ }^{3}$}

Rheumatoid arthritis (RA) patients and their healthcare providers frequently use the term 'flare' to describe periods of worsening disease activity. The term is not well defined and reflects a continuum of clinical experiences that is highly dependent on individual contextual factors. ${ }^{1-5}$ What constitutes a flare for one patient may not be the same as for another; what patients describe as a flare may be quite different from what a clinician considers significant; and the level at which symptoms become unmanageable may also vary from patient to patient and even in the same patient over time depending on disease activity, treatment effects and coping strategies. And while 'flare' is a commonly used term in English, it is not necessarily easily translated into other languages. ${ }^{2}$ With advances in RA treatment and improved outcomes, a careful study of the manifestations of flare is needed to identify patient-relevant elements that may encompass features beyond our current core set of outcome measures.

Early intensive treatment strategies that include combinations of non-biological and biological disease-modifying antirheumatic drugs are used to reach a treatment goal of remission or a very low disease activity state. ${ }^{6-10}$ By moving more patients to states of low disease activity, we now face new questions in clinical practice, namely how to taper therapy to maintain efficacy, to improve longerterm safety and potentially to lower costs. There is a limited evidence base from clinical practice and clinical trials to guide such decisions, partly because there is no standardised measure to assess significant disease worsening, or flare. By convention, an inversion of improvement criteria (driven

\footnotetext{
${ }^{1}$ Department of Rheumatology, Johns Hopkins University, Baltimore, Maryland, USA

2Internal Medicine, Rheumatology, Schlosspark-Klinik, Teaching Hospital Charite, Berlin, Germany ${ }^{3} \mathrm{VU}$ Medical Center, Amsterdam, The Netherlands
}

Correspondence to Clifton 0 Bingham III, Department of Rheumatology, Johns Hopkins University, Baltimore 21224, Maryland, USA; Clifton.bingham@jhmi.edu predominantly by physician-assessed variables) has often been adopted, ${ }^{11} 12$ with an assumption that the magnitude of relevant change for worsening and improvement will be similar, although studies suggest that clinically important thresholds of significant change differ for patients and physicians. ${ }^{13-15}$ Others have suggested that a physician's decision to change therapy represents a meaningful flare, but this represents only one end of a spectrum of disease worsening. ${ }^{1-5}$

In the past few years, there has been growing appreciation that incorporating patient-reported outcomes (PRO) into clinical trials and practice is necessary, ${ }^{16}$ and guidelines for the development of improved PRO have been released from consensus-based standards for the selection of health status measurement instruments, the US Food and Drug Administration and other regulatory bodies. ${ }^{17-21}$ Patient research partners have participated in the development of RA remission criteria, formulation of the RAID questionnaire to evaluate RA disease impact, multiple working groups within Outcome Measures in Rheumatology (OMERACT) and others. ${ }^{1} 24616$ 22-30 Through these efforts, patient-relevant domains concerning RA have been identified, which are not part of existing core sets of outcome measures, including fatigue and sleep. Moreover, it is increasingly recognised that clinical decision making should be shared between the patient and the clinician, balancing clinician 'knowledge' with patient 'experience'. Furthermore, existing PRO instruments (validated at a group level in clinical trials) have significant floor and ceiling effects at the individual patient level, including a major PRO in the core set, the health assessment questionnaire. There is thus a considerable opportunity to improve our understanding of the patient's perspective of illness to develop more inclusive and responsive outcome measures that will complement existing indices.

It is in this setting that Berthelot et a $\beta 1$ provide evidence that the patient's perspective of RA disease worsening or flare represents an experience extending beyond standard clinical outcome measures. Set in the context of the issues outlined above, the study demonstrates the type of approach necessary to develop a PRO to measure disease worsening that originates at the patient level. The study importantly reinforces that differences in opinion may exist between patients and clinicians in relevant variables.

Through parallel processes for RA patients and rheumatologists, a list of domains felt to be important in describing worsening disease activity was developed. Based on qualitative analysis of transcripts from more than 100 interviews with French RA patients, 10 domains were selected to represent the patient perspective of flare. Thirteen rheumatologists participated in a Delphi consensus process to identify domains of importance in describing flare, defined as a transient exacerbation of disease activity ultimately condensed to eight domains. Domains identified by both patients and clinicians were joint swelling, joint pain, sleep disturbance and analgesic use. It is notable that patients identified domains (fatigue, increased need for help, depression, social withdrawal and irritability) that were not identified by physicians. And some domains considered important by clinicians (morning stiffness, steroid dose and acute phase reactants) were not identified by patients. Domains from these parallel streams were merged into a final questionnaire for patients to determine if they had experienced worsening in domains, deemed important by patients and clinicians, compared with their last visit.

The questionnaire developed by Berthelot et $a^{\beta 1}$ is preliminary, and has not been validated against reference PRO or clinical disease parameters, nor has the English version undergone cognitive testing or validation. These are absolutely critical steps before any questionnaire is widely implemented. The instrument presented asks patients about their agreement with statements concerning worsening in various domains over 3 months or since the last clinical encounter. This construct has limitations as it relies on a considerable time period during which patients could have had more than one flare resulting in uncertainty of responses and issues related to recall bias, in which more distant events may not be remembered; it will not discern if symptom worsening may have been due to other non-RArelated features. While the questionnaire will potentially capture worsening that has taken place between scheduled visits, 
it will not necessarily capture flare as it is happening to trigger an evaluation. The questionnaire will largely capture information relevant to change rather than an absolute state of disease activity, yet both of these components may be important in evaluating flare. ${ }^{2} 35$ Moving forward it will be important to evaluate the duration and persistence of worsening as well as any interventions that may have been taken by the patient or clinician to manage the period of exacerbation.

Notwithstanding the above limitations, the work presented is a significant contribution concerning RA patients' perspectives of disease activity and worsening. The study is timely in its publication with other ongoing efforts, with qualitative results that parallel the recently reported work of the OMERACT group. In a qualitative study of RA flare led by Hewlett et a ${ }^{5}$ with 68 RA patients from five countries (UK, Canada, USA, Australia, Germany), many similar additional domains surfaced, including physical function, systemic features, cognitive dysfunction, participation and social withdrawal. In that study, the conceptualisation of patient 'self-management' (or failure thereof) as an important part of evaluating the magnitude and impact of disease worsening was introduced. It is important to note, however, that coping strategies and learned selfmanagement styles varied according to RA disease duration and severity (eg, earlier patients may be seen more frequently with healthcare providers providing more guidance compared with patients with longer-standing disease and less frequent visits managing more on their own) and from country to country depending on healthcare systems (eg, ability to access healthcare providers) and other cultural factors. In the study by Berthelot et al, ${ }^{31}$ the identified areas of 'asking for help' and 'analgesic use' may reflect this concept of self-management.

In the development of the rheumatoid arthritis impact of disease (RAID) score, ${ }^{30}$ created to assess overall RA disease activity rather than specifically flare, the discovery of patient-relevant domains was initiated with 10 RA patients from 10 European countries, and then evaluated in 100 additional patients (10 from each country). The domains ultimately included in RAID were similar to those elaborated by Berthelot et $a^{\beta 1}$ and Hewlett et $a l^{5}$ and included pain, fatigue, emotional wellbeing and sleep, but also physical function, coping and physical wellbeing. While RAID was not developed specifically for measuring worsening, its widespread adoption will quickly provide an evidence base to evaluate flares. That recurrent themes and domains have been identified in qualitative work through OMERACT, RAID and by Berthelot et a ${ }^{\beta 1}$ indicates a remarkable cross-cultural universality of the overall patient conceptualisation of RA disease activity. The studies of Berthelot et al, ${ }^{31}$ Hewlett et a ${ }^{5}$ and Gossec et $a^{\beta 0}$ all highlight the multiple dimensions of health-related quality of life that are impacted by RA from the patient's perspective, physically, emotionally and socially, and these extend far beyond the current core set PRO assessments of pain, physical function and fatigue.

Through the combined efforts of Berthelot et al,31 OMERACT and the RAID initiative a tremendous amount of information concerning disease worsening in RA will soon become available that will help to characterise the change in disease activity over time (for worsening and improvement) in additional patientrelevant aspects of disease. An RA flare $\mathrm{PRO}$ instrument requires validation in different clinical contexts (clinical practice, clinical trials), against legacy PRO, in relationship with other clinical variables and cross-culturally. In addition, the psychometric properties of individual questions will need to be evaluated, with a likely requirement for additional modifications and further testing as well as exploration of anchoring statements and recall periods. However, even the incorporation of these preliminary instruments into prospective observational and interventional studies will position us better to characterise minimally clinically important differences in terms of worsening that relate to changes in clinical parameters and result in a change in therapy. The importance of patient participation in developing and endorsing outcome measures is of increasing importance.

The studies mentioned above have included patients in various ways. When one considers an optimal level of involvement, patients would be seen as integral to all stages of outcome measure development and validation. In the study by Berthelot et al, ${ }^{31}$ while discovery of domains significantly included qualitative work from the patient level with subsequent testing in others, it is unclear whether patients were 'research partners' according to recently developed terminology. ${ }^{16}$ Both OMERACT and the European League Against Rheumatism (EULAR) have recognised this with the integration of patients at all levels of outcome measurement development. This begins at the conceptualisation phase, moves through discovery and validation, and culminates in the reporting of research findings. Patients have played substantial roles in the formulation of research goals, providing ongoing input to the research process, data analysis and interpretation (including review of transcripts from qualitative research), and presenting study data as abstracts, oral presentations and manuscripts. ${ }^{2} 41627$ The highest level of evidence to determine the 'truth' of a measure, especially one that is patient reported, would require the continuous involvement of patients at all levels of measure development and testing, recognising patients' voices as a requisite gold standard.

How might we envision the incorporation of a questionnaire to capture RA flare? A multidimensional instrument could be available for patients when they felt their disease was worsening. With the persistence of worsening symptoms of some sufficient magnitude for an appropriate period of time, this may trigger certain interventions (eg, increase non-steroidal anti-inflammatory drugs). With higher scores there may be a more intensive intervention (eg, add steroids); finally after failure of these interventions or worsening beyond a critical threshold there may be a trigger for a visit and consideration of changes in diseasemodifying antirheumatic drug therapy. At the time of a visit, patient self-report could be compared with clinical variables (swollen and tender joints, inflammatory markers, etc), and other comorbidities evaluated to determine if these may be responsible for symptom worsening. With a flare tool, patients could be provided with more information to guide their own RA management, in a manner similar to that incorporated in asthma management based on peak flow monitoring. ${ }^{32}$ Such a system may be helpful in identifying early worsening in patients who were in a low disease activity state or in remission to trigger earlier evaluation to determine whether to step up or reinitiate treatment to avoid more significant worsening or disability.

A great challenge for PRO that cover multiple domains of health is determining if changes are caused by RA disease activity or comorbidities (eg, an intercurrent infection). Without placement into the overall clinical context there is a danger of sole reliance on PRO data. This information should not supplant patient-physician interactions, but the availability of such data may, in fact, enhance communication between patients and healthcare providers and ultimately improve shared decision making. An ultimate 'definition' 
of flare used to trigger substantive treatment changes should not be an 'eitheror' between a PRO and clinical variables, rather this should be a composite 'loss-ofresponse' index that is more balanced in terms of perspectives.

The ongoing work concerning flare by several groups highlights the rich vocabulary used by RA patients to describe their disease experience. From these studies several important points emerge: (1) flare is a multifaceted and individualised experience; (2) current core outcome measures do not reflect this complexity; (3) our current 'definitions' of disease activity states based on composite indices and of disease worsening based on inversion of improvement criteria may require revision by more thoroughly incorporating the patient's perspective; and (4) there is a significant need to characterise and more accurately assess the patient's perspective of RA.

Some may focus on the challenges in studying flare and say that these are difficult if not impossible to measure. There are certainly some areas requiring further examination (eg, important identified domains without good measurement instruments, difficulty in reconciling physician and patient reports towards an aggregate measure, confounding of symptoms by comorbidities). While these are areas that will require more research, we should not see these as insurmountable obstacles; rather, we should remember the famous words of Sir William Osler, 'Listen to your patient, (s)he is telling you the diagnosis.' Indeed, it is likely that this is where our solutions are to be found.

Acknowledgements The authors acknowledge Dr Susan J Bartlett for her review and comments.

\section{Competing Interests None.}

Provenance and peer review Commissioned; externally peer reviewed.

Received 6 December 2011

Accepted 11 December 2011

Published Online First 9 February 2012

Ann Rheum Dis 2012:71:1107-1109.

doi:10.1136/annrheumdis-2011-200870

\section{REFERENCES}

1. Bingham CO, Pohl C, Alten R, et al. 'Flare' and disease worsening in rheumatoid arthritis: time for a definition. Int J Adv Rheumatol 2009;7:85-91.
2. Bingham CO III, Pohl C, Woodworth TG, et al. Developing a standardized definition for disease "flare" in rheumatoid arthritis (OMERACT 9 Special Interest Group). J Rheumatol 2009;36:2335-41.

3. Alten R, Pohl C, Choy EH, et al. Developing a construct to evaluate flares in rheumatoid arthritis: a conceptual report of the OMERACT RA Flare Definition Working Group. J Rheumatol 2011;38:1745-50.

4. Bingham CO III, Alten R, Bartlett SJ, et al. Identifying preliminary domains to detect and measure rheumatoid arthritis flares: report of the OMERACT 10 RA Flare Workshop. J Rheumatol 2011;38:1751-8.

5. Hewlett S, Sanderson T, May J, et al. 'I'm hurting, I want to kill myself': rheumatoid arthritis flare is more than a high joint count-an international patient perspective on flare where medical help is sought. Rheumatology (Oxford). 2012 Jan;51:69-76.

6. Felson DT, Smolen JS, Wells G, et al. American College of Rheumatology/European League against Rheumatism provisional definition of remission in rheumatoid arthritis for clinical trials. Ann Rheum Dis 2011;70:404-13.

7. de Wit MP, Smolen JS, Gossec L, et al. Treating rheumatoid arthritis to target: the patient version of the international recommendations. Ann Rheum Dis 2011;70:891-5.

8. Schoels M, Knevel R, Aletaha D, et al. Evidence for treating rheumatoid arthritis to target: results of a systematic literature search. Ann Rheum Dis 2010;69:638-43.

9. Smolen JS, Aletaha D, Bijlsma JW, et al. Treating rheumatoid arthritis to target: recommendations of an international task force. Ann Rheum Dis 2010;69:631-7.

10. Saag KG, Teng GG, Patkar NM, et al. American College of Rheumatology 2008 recommendations for the use of nonbiologic and biologic disease-modifying antirheumatic drugs in rheumatoid arthritis. Arthritis Rheum 2008:59:762-84.

11. Den Broeder AA, Creemers MC, van Gestel AM, et al. Dose titration using the Disease Activity Score (DAS28) in rheumatoid arthritis patients treated with anti-TNF-alpha. Rheumatology (Oxford) 2002;41:638-42

12. Rahman MU, Strusberg I, Geusens P, et al. Doubleblinded infliximab dose escalation in patients with rheumatoid arthritis. Ann Rheum Dis 2007;66:1233-8.

13. Leeb BF, Sautner J, Leeb BA, et al. Lack of agreement between patients' and physicians perspectives of rheumatoid arthritis disease activity changes. Scand J Rheumatol 2006;35:441-6.

14. Barton JL, Imboden J, Graf J, et al. Patient-physician discordance in assessments of global disease severity in rheumatoid arthritis. Arthritis Care Res (Hoboken) 2010;62:857-64.

15. Khan NA, Spencer HJ, Abda E, et al. Determinants of discordance in patient's and physician's rating of rheumatoid arthritis disease activity. Arthritis Care Res (Hoboken). 2 Nov 2011. doi:10.1002/acr.20685

16. de Wit MP, Berlo SE, Aanerud GJ, et al. European League Against Rheumatism recommendations for the inclusion of patient representatives in scientific projects. Ann Rheum Dis 2011;70:722-6.

17. Mokkink LB, Terwee CB, Knol DL, et al. The COSMIN checklist for evaluating the methodological quality of studies on measurement properties: a clarification of its content. BMC Med Res Methodo/ 2010;10:22.

18. European Medicines Agency (EMEA). Reflection Paper on the Regulatory Guidance for the Use of
Health-related Quality of Life (HROL) Measures in the Evaluation of Medicinal Products, 2005. http://www.ema.europa.eu/docs/en GB/ document_library/Scientific_guideline/2009/09/ WC500003637.pdf (accessed 11 Oct 2011).

19. FDA. Guidance for Industry - Patient-Reported Outcome Measures: Use in Medical Product Development to Support Labeling Claims. http://wwwfdagov/downloads/Drugs/ GuidanceComplianceRegulatoryInformation/ Guidances/UCM193282pdf (accessed 11 Oct 2011)

20. Critical Path Institute (cPATH). Patient Reported Outcomes (PRO) Consortium. http://wwwc-pathorg/ PROcfm (accessed 11 Oct 2011).

21. German Institute for Quality and Efficiency in Health Care (Institut für Qualität und Wirtschaftlickeit im Gesundheitswesen). Biotechnologisch hergestellte Arzneimittel in der Zweitlinientherapie bei der rheumatoiden Arthritis. https://www.iqwig. de/download/A10-01_Berichtsplan_Biologika Zweitlinientherapie bei rheumatoider_Arthritis.pdf (accessed 11 Oct 2011).

22. Kirwan JR, Newman S, Tugwell PS, et al. Progress on incorporating the patient perspective in outcome assessment in rheumatology and the emergence of life impact measures at OMERACT 9. J Rheumatol 2009:36:2071-6.

23. Kirwan JR, Hewlett SE, Heiberg T, et al. Incorporating the patient perspective into outcome assessment in rheumatoid arthritis-progress at OMERACT 7. J Rheumato/ 2005;32:2250-6.

24. Kirwan JR, Ahlmén $\mathrm{M}$, de Wit $\mathrm{M}$, et al. Progress since OMERACT 6 on including patient perspective in rheumatoid arthritis outcome assessment. $J$ Rheumatol 2005;32:2246-9.

25. Kirwan J, Heiberg T, Hewlett S, et al. Outcomes from the Patient Perspective Workshop at OMERACT 6. J Rheumatol 2003:30:868-72.

26. Gossec L, Dougados M, Rincheval N, et al. Elaboration of the preliminary Rheumatoid Arthritis Impact of Disease (RAID) score: a EULAR initiative. Ann Rheum Dis 2009;68:1680-5.

27. Kirwan JR, Fries JF, Hewlett SE, et al. Patient perspective workshop: moving towards OMERACT guidelines for choosing or developing instruments to measure patient-reported outcomes. J Rheumatol 2011;38:1711-15.

28. Kirwan JR, Tugwell PS. Overview of the patient perspective at OMERACT 10-conceptualizing methods for developing patient-reported outcomes. J Rheumato/ 2011;38:1699-701.

29. Sanderson T, Morris M, Calnan M, et al. Patient perspective of measuring treatment efficacy: the rheumatoid arthritis patient priorities for pharmacologic interventions outcomes. Arthritis Care Res (Hoboken) 2010;62:647-56.

30. Gossec L, Paternotte S, Aanerud GJ, et al. Finalisation and validation of the rheumatoid arthritis impact of disease score, a patient-derived composite measure of impact of rheumatoid arthritis: a EULAR initiative. Ann Rheum Dis 2011;70:935-42.

31. Berthelot JM, De Bandt M, Morel J, et al. A tool to identify recent or present rheumatoid arthritis flare from both patient and physician perspectives: The 'FLARE' instrument. Ann Rheum Dis 2012.

32. Gibson PG, Powell H. Written action plans for asthma: an evidence-based review of the key components. Thorax 2004;59:94-9. 